\title{
Ionospheric Traveling Vortex Generation by Solar Wind Buffeting of the Magnetosphere
}

\author{
MARGARET G. KIVELSON \\ Institute of Geophysics and Planetary Physics, University of California, Las Angeles \\ Department of Earth and Space Sciences, University of California, Las Angeles

\section{DAVID J. SOUTHWOOD} \\ Institute of Geophysics and Planetary Physics, University of California, Las Angeles \\ Department of P hysics, Imperial College of Science and Technology, London, UK.
}

\begin{abstract}
Traveling ionospheric vortices observed near the polar cusp boundary have been interpreted as signatures of impulsive reconnection (or flux transfer events) on the magnetopause, but neither the sense of motion nor the phase speed of the disturbances is consistent with the assumed generation mechanism. An altemative interpretation as the ionospheric signature of the response to fluctuating solar wind pressure can account for the reported features of the ionospheric perturbations. The solar wind pressure perturbation establishes vortical flow on magnetopause flux tubes and drives a guided shear wave along polar cusp field lines into the ionosphere. The guided shear wave carries the field-aligned currents needed to couple the magnetopause flow to ionospheric flow. The elementary form of the ionospheric disturbance in horizontal flow, electric field, and field-aligned current is dipolar in structure, with the flow near the center predominantly meridional and of the order of $100 \mathrm{~km} \mathrm{~s}^{-1}$ for pressure perturbations reported as typical in the solar wind. The phase velocity is $3-10 \mathrm{~km} \mathrm{~s}^{-1}$ in the east-west sense as inferred from mapping of a disturbance carried along the magnetopause at the magnetosheath flow velocity. A significant conclusion of this analysis is that the ionospheric signature of a traveling front across which pressure changes monotonically is a pair of vortices of opposite polarizations. A pressure pulse will produce two pairs of vortices, with the sense of polarization reversed in the trailing pair. Compressional perturbations of the boundary cannot produce isolated vortical flows in the ionosphere, although temporally very nonsymmetric perturbations could produce signatures that appear to have such symmetry.
\end{abstract}

\section{INTRODUCTION}

When the magnetospheric cavity is buffeted by changes in solar wind pressure, a variety of time series responses may ensue. In a previous paper [Southwood and Kivelson, 1990a], we showed that the response is a function of position; different responses can be observed at different locations in the cavity. In this paper we concentrate on the excitation of a particular type of response, the traveling ionospheric vortex. We outline a theory in which pressure perturbations at the boundary give rise to vortex motions in the ionosphere directly at the feet of the tubes comecting to the magnetopause. We show that localized traveling vortex motions are excited in the ionosphere not only in response to isolated pressure pulses, whether enhancements or depressions, but also in response to monotonic decreases or increases to new levels of external pressure.

Vortex motions of ionospheric plasma have been observed [Goertz et al., 1985; Lanzerotti et al., 1987; Friis-Christensen et al., 1988; McHenry et al., 1989; Glassmeier et al., 1989; Farrugia et al., 1989; Potemra et al., 1989] using data from ground magnetometer arrays at high latitude. The vortices often seem to occur in pairs; they travel with east-west propagation speeds usually of the order of $3-5 \mathrm{~km} \mathrm{~s}^{-1}$ [Friis-Christensen et al., 1988; Mchenry et al., 1989; Glassmeier et al., 1989] but even as large as $10 \mathrm{~km} \mathrm{~s}^{-1}$ [Potemra et al., 1989]. The material flow in the center of the paired vortices appears often to be roughly at right angles to the direction of travel. The ionospheric

\section{Copyright 1991 by the American Geophysical Union.}

Paper number 90JA01805.

0148-0227/91/90JA-01805\$05.00 disturbances have been interpreted as signatures both of flux transfer events (FTEs) [Russell and Elphic, 1978, 1979] and of pressure pulses [Elphic, 1988; Sibeck et al., 1989a, b] on the magnetopause. Various features of the ionospheric signatures have been used to distinguish among the suggested generation mechanisms, and below we present our reasons for thinking that pressure pulses on the magnetopause are the most plausible.

Our purpose, then, is to investigate the perturbation near the ionospheric ends of polar cusp flux tubes produced by pressure variations on the magnetopause. A pressure perturbation generates a compressional MHD signal which will propagate more or less isotropically in the magnetospheric cavity. Coupling to the ionosphere requires the generation of a field-aligned current system that is carried only by the field-guided Alfven mode as discussed in earlier papers [Kivelson and Southwood, 1990; Southwood and Kivelsom, 1990a, b]. In the present work, we examine specifically the generation of field-aligned currents on flux tubes connecting directly from the magnetopause to the high-latitude ionosphere. The restriction of attention to a portion of the ionosphere that links directly to the boundary enables us to follow the evolution of a perturbation as it propagates along flux tubes, and we can abandori the Laplace transform approach used in our previous paper [Southwood and Kivelson, 1990a].

\section{TWIN VORTICES}

A twin vortex of the fom detected by Friis-Christensen et al. [1988] is one of the simplest forms of low-frequency (long time scale) ionospheric-magnetospheric coupled flow. We illustrate a possible configuration of the vortex system in Figure 1. The balanced current flow into and out of the ionosphere yields a twodimensional dipolar perturbation pattem of the flow, and of the 


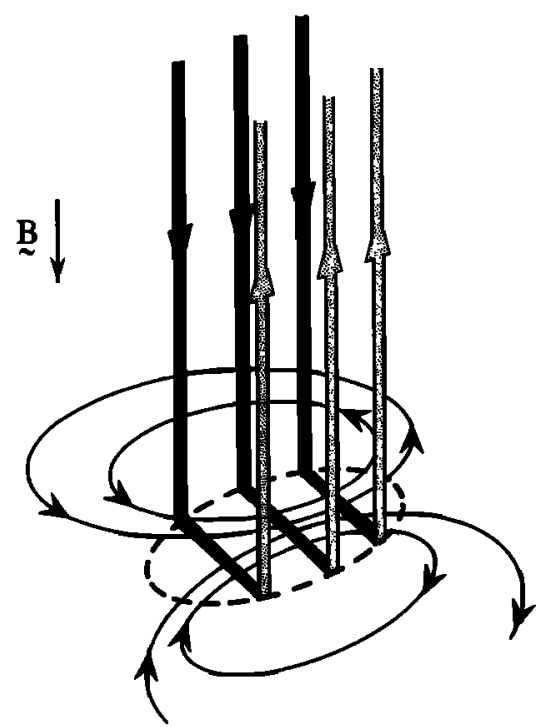

Fig. 1. Schematic illustration of the ionospheric perturbations produced by a balanced pair of distributed field-aligned currents flowing into, across, and out of the northern hemisphere ionosphere (shaded lines) within the flux tube bounded in the ionosphere by the dashed contour. The thin contours show flow streamlines (opposite to Hall current flow). The figure is based on a pattem proposed by Southwood [1987] as the ionospheric signature of a flux transfer event.

electric and magnetic perturbations. The figure is based on a pattem proposed by Southwood [1987] as the ionospheric signature of an FTE. Southwood was examining the consequences of moving a magnetospheric flux tube connecting the solar wind with the ionosphere through the surrounding plasma. In the FTE model, the plasma in the core of the tube (and thus in the center of the disturbance) is physically moving along the direction in which the entire pattem moves. However, the structures observed differ from the model as described above. Most of the reported cases reveal twin vortices separated in the east-west direction and traveling along the vortex separation direction. The flow in the center of the structure is predominantly meridional (north-south) and thus perpendicular to the direction of travel.

An additional characteristic of the reported ionospheric disturbances that we regard as critical is their speed. An FTE would create a traveling ionospheric signature through the material motion of plasma frozen onto a reconnected flux tube. In most relevant circumstances, material flow speeds are slower than the local sound speed. Typical sound speeds in the $E$ layer of the ionosphere are of the order of $1-2 \mathrm{~km} \mathrm{~s}^{-1}$. The reported vortex flow velocities of $3-10 \mathrm{~km} \mathrm{~s}^{-1}$ would require moving plasma at the feet of reconnected flux tubes at supersonic velocities and so are unlikely to correspond to material motion. A traveling disturbance on the magnetopause need not drag plasma through the ionosphere in order to impose perturbations; instead, the propagating disturbance connects successively to different flux tubes which "light up" as the front moves by. The angular velocity of a perturbation traveling along the equatorial magnetopause at a magnetosheath flow speed of roughly $100 \mathrm{~km}$ $\mathrm{s}^{-1}$ is about $0.1^{\circ} \mathrm{s}^{-1}$, which maps to approximately $5 \mathrm{~km} \mathrm{~s}^{-1}$ at the polar cusp ionosphere. This velocity argument suggests strongly that most observed vortices in the dayside ionosphere are the ground signatures of propagating disturbances on the boundary.
Let us examine how a twin vortex flow pattern is imposed on the ionosphere by a change in the pressure equilibrium conditions at the high-altitude magnetopause. The coupling of the plasma motions along the flux tube requires the presence of field-aligned currents, and Figure 1 shows that in the ionosphere these currents are colocated with the centers of the flow vortices [Glassmeier et al., 1989]. In fact, where the background field, $B$, is approximately vertical and the Pedersen conductivity, $\sigma_{\mathrm{P}}$, is horizontally uniform, one has

$$
-\nabla \cdot J_{\|}=\nabla \cdot J_{\perp}=\nabla \cdot\left(\sigma_{P} E\right)=-\sigma_{P}(\nabla \times u) \cdot B
$$

Here $\left.\mathbf{J}=\mathbf{J}_{\perp}, \mathfrak{J}_{\|}\right)$is the current density expressed in terms of components perpendicular and parallel to $B, u$ is the fluid velocity, and $\mathbf{E}$ is the perturbation electric field $(=-\mathbf{u} \times \mathbf{B})$. Equation (1) shows that the divergence of the horizontal current (as well as the related variation of parallel current) is proportional to the field-aligned component of the vorticity, $\Omega_{\|}=\nabla_{\perp} \times \mathbf{u}$. Thus twin vortex flow necessarily implies a pair of field-aligned currents directed into and out of the ionosphere.

\section{EXCTTATION OF THE IONOSFIERE BY COMPRESSIONAL AND SHEAR MAGNETOSPHERIC MOTIONS}

The ionosphere can be thought of for our purposes as a thin conducting boundary. At first glance, one might not expect the ionosphere to respond at all to a change in pressure from outside the magnetosphere. Compression (or decompression) of the magnetosphere at high altitude will launch a compressional (fast) hydromagnetic wave propagating through the system. As is pointed out by Kivelson and Southwood [1988] but also is implicit in earlier work [e.g., Nishida, 1964; Hughes and Southwood, 1974], a compressional wave incident on the ionosphere from the magnetosphere is almost perfectly reflected. In contrast, the Alfven (or shear) mode does penetrate the ionosphere. Some wave energy is absorbed by the ionosphere, and the reflection coefficient is proportional to $\Sigma_{\mathbf{p}}$, the heightintegrated Pedersen conductivity. Thus, in order to understand the ionospheric perturbation, one needs to examine how compressional perturbations couple to shear motions.

\section{EXCTIINg SHEAR Motions AT THE MAGNETOPAUSE}

One means for conversion of compressional energy into shear motions is the mode coupling introduced by inhomogeneity, as Southwood and Kivelson [1990a] point out. Mode coupling occurs when the scale of variation in the disturbance is comparable to the scale of variation of the background field. Such coupling seems likely to give rise to the localized magnetic oscillations detected in ground magnetometer records in association with pressure changes in the solar wind by Farrugia et al. [1989] (see also Southwood and Kivelson [1990a], but it does not seem a likely explanation of the isolated and fairly localized high-latitude vortex signatures. Here we focus on a more likely explanation, the direct generation of vorticity at the foot of the polar cusp driven by boundary pressure perturbations and their associated field-aligned currents.

Let us consider the imposition of a pressure perturbation, $\Delta p(s, \varphi, t)$, on the boundary of the magnetosphere. Here $\varphi$ is azimuth (or longitude) and $s$ represents distance along the field line measured from the equator, say, at the magnetopause. Across the boundary, pressure is balanced. Ignoring the interior plasma pressure and assuming small field perturbations, the field pressure in the immediate vicinity of the boundary changes by an 
amount, $\mathbf{B} \cdot \mathbf{b} / \mu_{0}$, where

$$
\Delta \mathrm{p}(s, \varphi, t)=\frac{B \cdot b(s, \varphi, t)}{\mu_{0}}
$$

Elsewhere there will be a magnetic perturbation that satisfies

$$
\mu_{0 \rho} \frac{\partial u}{\partial t}=(\nabla \times b) \times B
$$

The perturbations that we are interested in are imposed on a time scale long compared with the time it takes a fast compressional wave to propagate through the interior of the magnetosphere. For example, the velocity characterizing the passage of a pressure perturbation on the boundary is of the order of the magnetosheath flow speed and considerably smaller than the Alfven velocity in the magnetosphere. This means that the compressional perturbation is imposed quasi-statically and takes the form of a surface wave decaying inward from the magnetopause [Southwood and Kivelson, 1990a].

Whereas the compressional component adopts a global quasistatic configuration, shears in the flow, parallel vorticity, fieldaligned currents and other physical quantities associated with the Alfven mode are strictly guided by the field. In the next section, we point out that a compression at the boundary not only launches a compressional wave but also excites a sheet of vorticity at the boundary which generates an Alfvern wave. It is the development and propagation of this disturbance that we next investigate.

We introduce a field-aligned coordinate system $(\Phi, \phi, s)$ based on the background field. The Euler potentials $\Phi$ and $\phi$ are constant along field lines and label the meridional and longitudinal directions, respectively. They can be chosen such that

$$
\mathbf{B}=\nabla \Phi \times \nabla \phi
$$

[e.g., Southwood, 1977; Southwood and Kivelson, 1989]. Unit vectors perpendicular to the field can be written in terms of the scale factors $h_{\Phi}$ and $h_{\phi}$ as

$$
\begin{aligned}
& \hat{\Phi}=h_{\boldsymbol{\Phi}} \nabla \Phi \\
& \hat{\phi}=h_{\phi} \nabla \phi
\end{aligned}
$$

with $\hat{\boldsymbol{\Phi}} \cdot \hat{\phi}=0$ and $h_{\Phi} h_{\phi}=B^{-1}$

The time derivative of (3) in conjunction with Faraday's law may be used to write equation (3) as a wave equation. The form may be inferred in the manner described by Singer et al. [1981]:

$$
\begin{gathered}
{\left[\mu_{0} \rho \frac{\partial^{2} u_{\Phi}}{\partial t^{2}}-h_{\Phi}{ }^{-1} \mathbf{B} \cdot \nabla h_{\Phi}{ }^{2} \mathbf{B} \cdot \nabla\left(u_{\Phi} / h_{\Phi}\right)\right]} \\
=-\mathrm{B}^{2} \frac{1}{h_{\Phi}} \frac{\partial^{2}}{\partial t \partial \Phi}\left(b_{\|} / \mathrm{B}\right)
\end{gathered}
$$

The $\phi$ component of the velocity satisfies the equivalent equation with $\Phi \rightarrow \phi$. The scale factors complicate the result in the general case, but in a quasi-dipolar field, the approximation that $h_{\Phi} \approx h_{\phi}$ may be introduced. This approximation, which allows us to set $h_{\Phi}=h_{\phi}=(B)^{-1 / 2}$, simply requires that distances between field lines vary with $s$ independent of perpendicular direction. Provided the source pressure varies with $s$ more rapidly than does the background field, equation (4) and the corresponding equation for $u_{\phi}$ then become

$$
\begin{gathered}
{\left[\frac{\mu_{0} \rho}{\mathrm{B}^{2}} \frac{\partial^{2}\left(\mathrm{u}_{\perp}\right)}{\partial t^{2}}-\mathrm{B}^{-1 / 2(\mathrm{e} \cdot \nabla)^{2}\left(\mathrm{~B}^{1 / 2} \mathrm{u}_{\perp}\right)}\right]} \\
=-\frac{\partial}{\partial t} \nabla_{\perp}\left(b_{\mid} / \mathrm{B}\right)
\end{gathered}
$$

wherc $e=B / B$ and the subscript on the $u$ and on the $\nabla$ operator denotes either the $\Phi$ or the $\phi$ component.

If the spatial variation of the perturbation pressure perpendicular to $B$ is more rapid than the variation of the background field, the solutions can be made up of separable functions of $s$ and the perpendicular coordinates, $\Phi$ and $\phi$. Moreover, it also follows that as the r.h.s. of (5) is the twodimensional gradient of a scalar quantity, any solution for $u$ will have zero parallel curl everywhere except at the boundary. At the boundary, however, a pressure change will induce motions not only inward or outward (for convenience, we shall refer to this as meridional motion) but also parallel to the plane of the boundary (henceforth referred to as the azimuthal direction). The displacements and the streamlines of the magnetospheric flow are illustrated in Figure 2. The purely inward or outward portion of the plasma displacement is compressional and, like compressional waves in a uniform plasma, drives perpendicular currents. The perpendicular perturbation currents on the magnetopause in the vicinity of the front correct the Chapman Ferraro currents to satisfy the new equilibrium conditions. Those currents close either at high latitudes on the magnetopause or
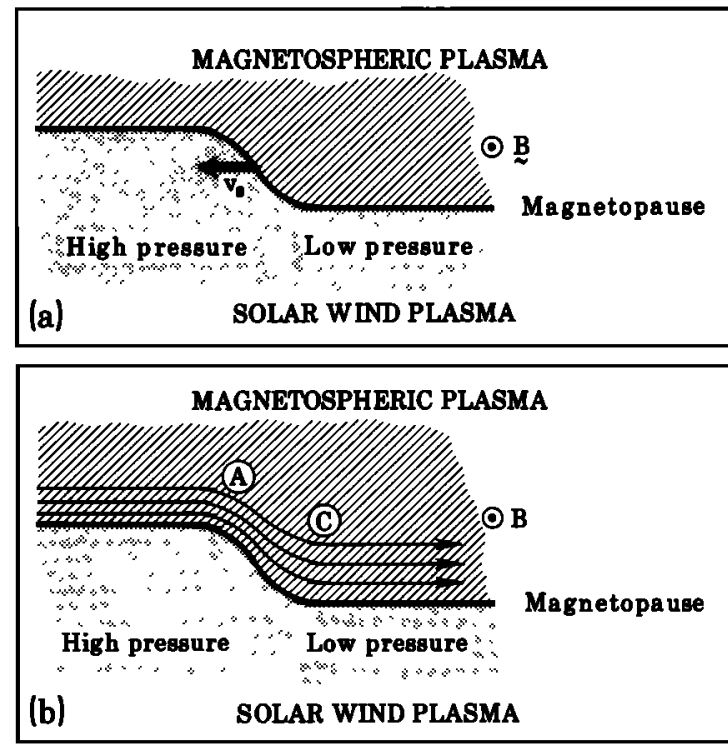

Fig. 2. Schematic of a pressure perturbation on the nearequatorial magnetopause. Figure $2 a$ is shown in the rest frame of the magnetosphere with the front across which the pressure decreases propagating to the left with velocity $\mathbf{v}_{\mathrm{s}}$. Figure $2 b$ is shown in the rest frame of the front and shows streamlines of the magnetospheric plasma. This illustrates how vorticity develops as a consequence of the propagation of the front along the boundary. Near $\mathbf{C}$ in the diagram, the parallel vorticity is positive and the field-aligned current flows into the equator. Near $A$ in the diagram, the parallel vorticity is negative and the fieldaligned current flows away from the equator. 
within the magnetosphere (transverse to the background field) depending on the specific form of pressure change envisioned. On the other hand, the azimuthal propagation of the front imposes shears in the flow which, like shear Alfvén waves in a uniform plasma, drive field-aligned currents. These are the currents that couple into the ionosphere [Southwood and Kivelson, 1990b]. Thus, it is clear that our interest must focus principally on the azimuthal perturbations of the flow.

The azimuthal component of the flow satisfies

$$
\begin{gathered}
{\left[\frac{\mu_{0} \rho}{\mathrm{B}^{2}} \frac{\partial^{2}}{\partial t^{2}}-(\mathrm{e} \cdot \nabla)^{2}\right]\left(\mathrm{B}^{1 / 2} u_{\phi}\right)=-\mathrm{B} \frac{\partial^{2}}{\partial \phi \partial t}\left(\mathbf{b} \cdot \mathbf{B} / \mathrm{B}^{2}\right)} \\
=-\mathrm{B} \frac{\partial^{2}}{\partial t \partial \phi} \mu_{0} \Delta p(s, \phi, t) / \mathrm{B}^{2}
\end{gathered}
$$

where the final form of the right-hand side applies at the boundary (cf. equation (2)).

The equation governing the azimuthal component of the flow immediately adjacent to the boundary (the second form of equation (6)) is a guided wave equation for $\left(B^{1 / 2} u_{\phi}\right)$ bdy, where the subscript designates the location on the magnetopause boundary. The function on the r.h.s. is completely specified once the pressure perturbation imposed at the boundary is defined. The significance of the equation is clearest if there is a frame in which the front is at rest (see Figure 2). If the front has an azimuthal phase velocity $U$, the time derivative on the right hand side of (7) becomes $\partial / \partial t=\mathrm{U} \cdot \nabla=\mathrm{U}\left(\mathrm{h}_{\varphi}{ }^{-1}\right) \partial \partial \varphi$, and with this substitution, the equation adopts the form

$$
\begin{aligned}
& {\left[\frac{\mu_{0} \rho}{\mathrm{B}^{2}} \frac{\partial^{2}}{\partial t^{2}}-(\mathrm{e} \cdot \nabla)^{2}\right]\left(\mathrm{B}^{1 / 2} u_{\phi}\right) \mathrm{bdy}} \\
& \quad=-\mathrm{B} U h_{\phi}{ }^{-1} \frac{\partial^{2}}{\partial \phi^{2}}\left[\mu_{0} \Delta \varphi(s, \phi, t) / \mathrm{B}^{2}\right]
\end{aligned}
$$

The field lines lying along both the low-latitude and polar sections of the magnetopause leave the boundary in the cusp regions of the magnetosphere (see the sketch in Figure 3 ). It follows that the flow perturbation represented by $\left(B^{1 / 2} u_{\varphi}\right)$ bdy traveling in the guided shear wave mode can leave the boundary at the cusp and propagate down the field to the ionosphere.

During a general compression of the magnetosphere, compressional signals may be present at all latitudes on the polar cusp flux tubes that form the dayside magnetopause. The actual coupling from the boundary into the cusp may be complicated. We consider solely the perturbations produced by changes in pressure on the low-latitude magnetopause. Perturbations driven on near-equatorial portions of boundary field lines propagate to higher-latitude parts that have left the boundary. At the point where field lines leave the boundary, we shall assume that transverse motions inside the magnetosphere are continuous with respect to the coordinate $s$. Once the wave has left the boundary, the wave motion of the plasma in the meridian must be continuous in the direction normal to the background field. As noted above, the flow fields in the traveling signals adopt a pattem like that of a surface wave. The amplitude of the meridional motion will be peaked at the cusp field line but will extend on either side of the tube in the meridian decaying in each direction. The azimuthal flow is imposed by the boundary perturbation only for $s<s_{0}$ in Figure 3. For $s>s_{0}$, the propagating wave carries a field-aligned current that imposes a

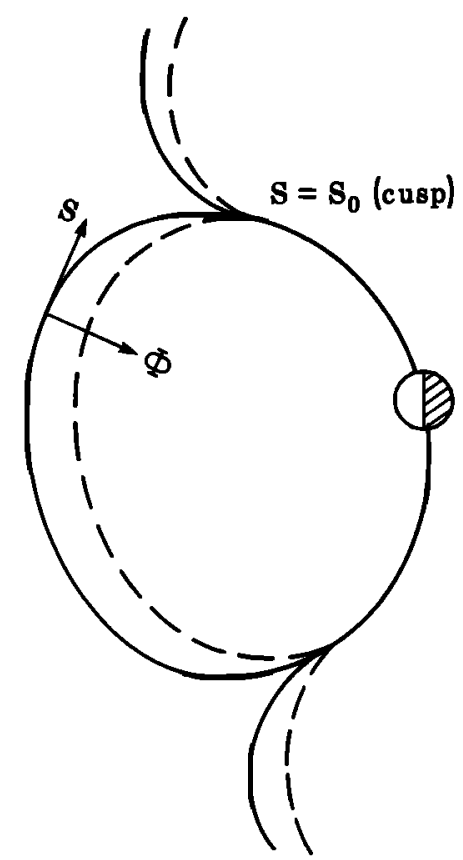

Fig. 3. Schematic illustration of polar cusp field lines linked from the dayside boundary to the ionosphere. The solid and dashed curves represent the same field line before and after an increase of solar wind dynamic pressure. The field-aligned curvilinear coordinate system has $s$ parallel to the local direction of the unperturbed field and $\Phi$ along the meridional normal direction. At the equator we set $s=0$, and $s_{0}$ is the point at which the field line leaves the boundary. The field lines lying along both the low-latitude and polar sections of the magnetopause leave the boundary at $s_{0}$ and continue down to the ionosphere through the interior of the magnetosphere.

$u_{\phi}$ oppositely directed on the two sides of the field line in the $\Phi$ direction, thereby imposing a shear, which we write as $\left[u_{\varphi}\right]$, of amplitude $2\left(u_{\varphi}\left(s_{0}\right)\right)_{\text {bdy }}$ across the cusp field line. Beyond the cusp, the azimuthal shear thus created is carried down the flux tube as a freely propagating Alfvén wave, i.e. the shear, satisfies an equation like (7) without the source term:

$$
\left[\mu_{0} p \frac{\partial^{2}}{\partial t^{2}}-(\mathrm{B} \cdot \nabla)^{2}\right] \mathbf{B}^{1 / 2}\left[u_{\phi}\right]=0
$$

Equations (7) and (8) show that the shear flow induced by the pressure change obeys a one-dimensional wave equation both at the boundary and between the cusp and the ionosphere. The wave motion is forced at the boundary itself [cf. the r.h.s. of (7)]. Elsewhere on the flux tube, there should be no forcing or source term. One may formally write the form of the solution in terms of the source function on the right of (7) using Green's functions [Morse and Feshbach, 1953]. Evidently, the propagation along the field will introduce a delay between source and responses in the ionosphere corresponding to the travel time of the wave.

The variation of the pressure distribution in time and space determines the precise form of the wave fields. In Figure 4 we sketch the type of source function set up by a traveling compressional pressure front of the sort illustrated in Figure 2. The source function is proportional to the second derivative of the pressure in the azimuthal direction. The sketch shows that the source of azimuthal flow at the boundary changes sign across the region of changing pressure. As the gradient in azimuthal 
pressure increases, the plasma is pushed azimuthally in one direction, and as the gradient decreases in the latter half of the pulse, the plasma moves in the opposite sense, creating the dipolar form described earlier.

Any pressure front imposed on the magnetosphere from outside is likely to move at a substantial speed. As a guide, let us assume that it moves at a speed comparable to the velocity of the magnetosheath plasma, $\sim 10^{2} \mathrm{~km} \mathrm{~s}^{-1}$. The Alfvén speed inside the boundary (of the order of $10^{3} \mathrm{~km} \mathrm{~s}^{-1}$ ) is likely to be larger than the sheath speed and so the shear motion is rapidly transmitted along the field, smearing out phase structure imposed by the travel of the front along the direction of the interior field tending to align phase fronts along the field. However, the phase structure perpendicular to the interior field direction is better maintained. It follows that the signals launched from the cusp down to northem and southern ionospheres will retain a scale of azimuthal variation comparable to that of the source pressure pulse. Say this scale is $N R_{E}$. If the propagation speed is $100 \mathrm{~km}$ $\mathrm{s}^{-1}$ in the equatorial plane, the time scale to launch the pulse is about $60 N$ seconds. For small $N$ (less than 3 or 4 ), this time is likely to be less than or of the order of the travel time for the pulse from the cusp to the ionosphere (also a few minutes). In this case the signal may lag behind the source as it travels down the field line. Then the pulse reflects off the ionosphere and travels back into the magnetosphere on different flux tubes, and the lag between the boundary disturbance and the ionospheric response is an Alfvén wave travel time, again of the order of a few minutes. One can imagine a single pressure front imposing in this manner a train of vortices separated by the travel time for the shear wave between hemispheres, but such signals might be difficult to detect as different portions of the input signal could generate disturbances that would interfere with each other. The effect would be enhanced if the Alfven velocity were unusually low (say of the order of $400 \mathrm{~km} \mathrm{~s}^{-1}$, as it would be if low-latitude boundary layer plasma with density of the order of $10 \mathrm{~cm}^{-3}$ and B $=60$ n'T were present).

When the time scale for imposition of the pulse is longer ( $N$ much larger than 3 or 4 ), the signals will reflect between

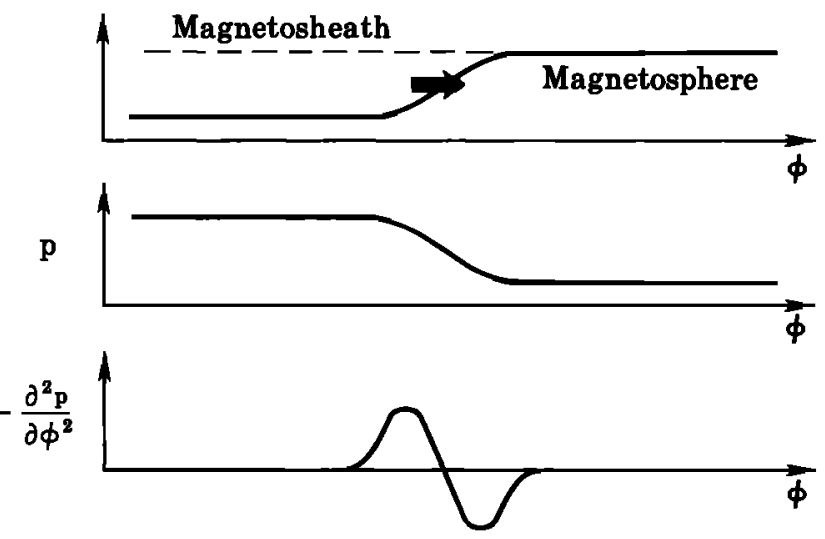

Fig. 4. Schematic of the boundary perturbations produced by an increase of solar wind dynamic pressure. The top panel shows the boundary between the magnetosheath and the magnetopause in the $\Phi-\phi$ plane in the presence of a traveling compressional wave moving in the $\phi$ (azimuthal) direction. The middle panel shows the variation of the pressure on the boundary as a function of $\phi$. The second derivative of the pressure is sketched in the bottom panel, which shows the bipolar form of the source function that drives field-aligned currents. magnetopause and ionosphere on a single flux tube and waves standing along $B$ will be locally excited. In principle, the boundary disturbance could be modified by contributions from waves reflected back from the ionosphere, but such an effect is neglected in this treatment. For the events reported by Sibeck et al. [1989a], the time scale for solar wind pressure pulses was about $8 \mathrm{~min}$, so the case of $N>4$ is relevant. The boundary perturbation is imposed on the system in the form of a forced oscillation at a frequency low enough that the system tracks the perturbing force directly.

The flow in the wave is necessarily vortical and thus will penetrate when it reaches the ionosphere. As equation (1) shows, it will set up field-aligned currents into and out of the ionosphere on the tube which maps to the boundary, i.e., the tube across which the discontinuity in azimuthal flow occurs. The polarity of the field-aligned currents will be alike in the two hemispheres, that is, either inward on the leading side and outward on the trailing side, or vice versa. The dipolar nature of the source deduced earlier means that a simple pressure increase (or decrease) sets up a twin vortex configuration in the ionosphere. A pulse of pressure (increase then decrease or vice versa) gives rise to a quadrupolar source configuration and a similarly complex arrangement of field-aligned currents. Evidently, the form of the signal can be very much modified if pressure sources of greater complexity are present on the boundary or if they are distributed along portions of the boundary field lines away from the equator.

\section{OBSERVATIONS AND DISCUSSION}

The most detailed reports of traveling ionospheric vortices are those of Goertz et al. [1985], Friis-Christensen et al. [1988], McHenry et al. [1989], and Glassmeier et al. [1989]. In each of these reports, the evidence for vortical motion is clear and the resemblance to the pattem of fields predicted to be set up by the passage of a flux transfer event at high altitude [Southwood, 1987] has been remarked. In the events of the Goertz et al. study, the sense and speed of motion of the signatures recorded are generally consistent with the Southwood FTE model. In the other cases above, they are not. The origin of these latter examples of vortices in flux transfer events is also called into question as there does not appear to be any clear correlation with southward components of the interplanetary magnetic field [McHenry et al., 1989; Glassmeier et al., 1989].

An alternate interpretation, related to a conjecture put forward by Elphic [1988], is treated theoretically in this paper. We suggest that the source of the vortical flows in the ionosphere is specifically the azimuthal flow imposed at the magnetopause by the passage of a pressure front in the solar wind. The flow imposed at the boundary excites a dipolar field-aligned current that drives a vortical flow in the ionosphere. The ionospheric pattern travels east-west along the polar cusp boundary at a speed comparable to the mapped magnetosheath speed, i.e., faster than the mean speed of the plasma in the ionosphere. The central flow is north-south, and thus it follows that the dipole orientation is primarily in the azimuthal direction, exactly as described by Glassmeier et al. [1989].

When the source dipole is aligned along the plane of the boundary and travels azimuthally, the pair of field-aligned currents are separated principally in the azimuthal direction. It follows that in the center of the twin vortex, the flow is at right angles to the direction of motion. The propagation speed of a few hundred kilometers per second at the magnetopause 
corresponds to a speed of less than or of the order of $10 \mathrm{~km} \mathrm{~s}^{-1}$ in the ionosphere, which fits well with the observations of vortices reported by Friis-Christensen et al. [1988], Mchenry et al. [1989], Glassmeier et al. [1989], Farrugia et al. [1989], and Potemra et al. [1989].

On order of magnitude grounds, the maximum velocity that can be imposed on plasma in the equatorial plane is of the order of $\left(\Delta p / \rho_{\text {sheath }}\right)^{1 / 2}$. For the slowest perturbations, a quasiequilibrium can be established between the equator and the ionosphere, in which case the flow would map as the square root of $B$, thus decreasing by a factor of order 30 . However, for more rapidly imposed pressure changes, the mapped velocity would be lower than this by up to a factor of about 10 (the ratio that would apply for a standing Alfven wave). It follows that the maximum amplitude of the velocity in the ionosphere could be of the order of the phase velocity but it will nomally be less than that by up to a factor of about 10 .

Although our model follows Elphic's [1988] conjecture in assuming that changes in solar wind dynamic pressure drive the ionospheric vortices, we diverge from his schematic description of the mechanism in two significant ways. Elphic stresses the compressional part of the perturbation and the associated changes in the location of the boundary. He interprets the ionospheric flows by mapping the radial displacements of the magnetopause into the polar cap. However, the compressional perturbations do not by themselves drive the field-aligned currents needed to set the ionosphere into motion, so we direct our attention to the azimuthal flows that are associated with the boundary displacement and that drive field-aligned currents into the ionosphere. Because Elphic draws a direct link between the motions of the boundary and the ionosphere, he requires a nonmonotonic pressure variation to account for the existence of a pair of vortices. Our interpretation, on the other hand, associates a twin vortex directly with a monotonic pressure variation. For nonmonotonic pressure variations, we would expect quadrupolar or other flow patterns to develop.

It should be noted that the relation between field-aligned currents in the ionosphere and dynamic pressure perturbations in the solar wind was considered many years ago by Tamao [1964]. His model, much ahead of its time, proposed the development of vortical flows in the ionosphere through the conversion of compressional to transverse waves. However, his dual fieldaligned currents separate and flow along opposite flanks of the magnetosphere, producing isolated vortices on each side. Our model differs from this picture by generating the shear Alfvén perturbations directly at the boundary instead of through mode conversion within the magnetosphere.

Assuming that impulsive events at high latitudes are signatures of FTEs on the magnetopause, Bering et al. [1988] described the signatures predicted for the electric field at balloon altitudes using two different models, the Southwood [1987] current dipole model (see Figure 1) and the coaxial current model implied by multiple X line recomection [Lee and $F_{u}, 1985$; Lee et al., 1988]. In their analysis, they assumed from the start that the patterns were moving poleward over the balloon. The type of phenomenon discussed in this paper could well make signatures like those discussed by Bering et al.; however, in any current distribution models derived from the theory presented here, the phase motion and the dominant plasma motion in disturbances are separate and can be orthogonal. The phase motion is predicted to be east-west with anti-solar speeds of the order of 3 to $10 \mathrm{~km} \mathrm{~s}^{-1}$ in the ionosphere. The plasma motion is vortical of amplitude less than the phase motion and in the simplest cases is north-south in the center of events.

Many of the ionospheric signatures that have been tentatively associated with FTE or other boundary phenomena [e.g., Lanzerotti et al., 1987] appear as isolated spikes that relate readily to a single vortex rather than the twin vortices discussed here. We suggest that boundary pressure variations are capable of producing single vortex patterns only accidentally as a result of asymmetries of the pressure variations. If the source function has a very nonsymmetric form as would occur if the pressure rises rapidly and levels off slowly, the field-aligned currents flowing into the ionosphere would create a very unsymmetrical electric field and flow pattern. The currents at the leading edge of the impulse would be confined spatially, thus producing clearly identifiable signatures, whereas the currents at the trailing edge of the pattern would be distributed azimuthally over much larger distances at low intensity. In such an asymmetric distribution, measurements of ionospheric flow and ground magnetic perturbations might fail to respond to the distributed closure currents. The pattern could mimic some of the signatures of a single vortex.

More recently, Lanzerotti et al. [1990] have reported on magnetic "impulse" signatures observed at conjugate stations and report reasonably good conjugacy, which is consistent with the model of their generation put forward here. In some of these more recently reported cases, the signatures observed appear to be driven by bipolar current patterns of the sort we discuss, but some of the events are ambiguous. The observation of impulses does not correlate with the orientation of the solar wind magnetic field. Lanzerotti et al. cannot determine whether all the impulses are bipolar or whether there can be unipolar impulses as well.

Although material motion and phase motion are not directly connected, it is important to note that in a disturbance with a surface mode structure (as applies in this case), there is a net nonlinear momentum transport in the direction of phase travel [Southwood, 1979]. A further point that needs to be noted is that our theory predicts vortex generation for any change in solar wind pressure (increase or decrease). It follows that increases and decreases in solar wind pressure, i.e., general buffeting. contribute to anti-solar momentum transfer into the magnetospheric cavity.

\section{SUMMARY}

Our study has been directed at explaining traveling vortical motions recorded in the ionosphere and in particular to examining pressure changes imposed at the magnetopause as a source. A previous paper had pointed out the variety of potential responses that a change in pressure at the magnetopause could produce. Here we have focussed attention on the direct transmission of pressure-induced motion to the ionosphere by means of the magnetohydrodynamic Alfvén mode, which naturally gives rise to field-aligned current and vortex motion. We conclude that (1) azimuthal flows are excited at the equatorial magnetopause by an externally applied pressure pulse, (2) the azimuthal flow gives rise to a field-aligned current system and flow vortex pattern similar to what is observed in the ionosphere, (3) a single rise (or fall) in pressure (i.e., a pressure front) gives rise to a pair of vortices of opposite sense, (4) a single pulse (rise and fall) gives rise to two pairs of vortices (a quadrupolar configuration), and (5) the model is least capable of explaining isolated current surges (unipolar current). It is important to note 
that signatures of single current surges may have been seen [Lanzerotti et al., 1987, 1990], but it is difficult to confirm the symmetry of the driving currents unless the spatial coverage of the ground observing stations is comparable with the scale of the structures. Should it become certain that unipolar field-aligned currents give rise to some of the impulses, we would be reluctant to attribute them to pressure pulses originating in the solar wind.

Acknowledgments. We thank R. C. Elphic for raising some interesting questions about a previous version of this paper. This work was supported by the Division of Atmospheric Sciences of the National Science Foundation under grant ATM 86-10858 and ATM 89-13342. UCLA Institute of Geophysics and Planetary Physics Publication 3318.

The Editor thanks K.-H. Glassmeier and D. G. Sibeck for their assistance in evaluating this paper.

\section{REFERENCES}

Bering, E. A., III, J. R. Benbrook, G. J. Byme, B. Liao, J. R. Theall, L. J. Lanzerotti, C. G. Maclennan, A. Wolfe, and G. L. Siscoe, Impulsive electric and magnetic field perturbations observed over south pole: Flux transfer events?, Geophys. Res. Lett., 15, 1545, 1988.

Elphic, R. C., Multipoint observations of the magnetopause: Results from ISEE and AMPTE, Adv. Space Res., 8, 223, 1988.

Farrugia, C. J., M. P. Freeman, S. W. H. Cowley, D. J. Southwood, M. Lockwood, and A. Etemadi, Pressure driven magnetopause motions and attendant response on the ground, Planet. Space Sci., 37, 589, 1989.

Friis-Christensen, E., M. A. McHenry, C. R. Clauer, and S. Vennerstrom, Ionospheric travelling convection vortices observed near the polar cleft: A triggered response to sudden changes in the solar wind, Geophys. Res. Lett., 15, 253, 1988.

Glassmeier, K.-H., M. Honisch, and J. Untiedt, Ground-based and satellite observations of traveling magnetospheric convection twin vortices, J. Geophys. Res., 94, 2520, 1989.

Goert, C. K., E. Nielsen, A. Korth, K. H. Glassmeier, C. Haldoupis, P. Hoeg, and D. Hayward, Observation of a possible ground signature of flux transfer events, J. Geophys. Res., 90, 4069, 1985.

Hughes, W. J., and D. J. Southwood, The effect of the atmosphere and ionosphere on magnetospheric micropulsation signals, Nature, 249, 493, 1974.

Kivelson, M. G., and D. J. Southwood, Hydromagnetic waves and the ionosphere, Geophys. Res. Lett., 15, 1271, 1988.

Kivelson, M. G., and D. J. Southwood, Magnetopause pressure pulses as a source of localized field-aligned currents in the magnetosphere, in Physics of Magnetic Flux Ropes, Geophys. Monogr. Ser., vol. 58, edited by C. T. Russell, E. R. Priest, and L. C. Lee, p. 619, AGU, Washington, D. C., 1990.

Lanzerotti, L. J., R. D. Hunsaker, D. Rice, L.-C. Lee, A. Wolfe, C. G. Maclennan, and L. V. Medford, Ionosphere and groundbased response to field-aligned currents near the magnetospheric cusp region, J. Geophys. Res., 92, 7739, 1987.

Lanzerotti, L. J., A. Wolfe, N. Trivedi, C. G. Maclennan, and L. V. Medford, Magnetic impulse events at high latitudes: Magnetopause and boundary layer plasma processes, $J$. Geophys. Res., 95, 97, 1990.
Lee, L.-C., and Z. F. Fu, A theory on magnetic flux transfer at the Earth's magnetopause, Geophys. Res. Lett., 12, 105, 1985.

Lee, L.-C., Y. Shi, and L. J. Lanzerotti, A mechanism for the generation of cusp region hydromagnetic waves, $J$. Geophys. Res., 93, 7578, 1988.

McHenry, M. A., C. R. Clauer, E. Friis-Christensen, and J. D. Kelly, Observations of ionospheric convection vortices: Signatures of momentum transfer, Adv. Space Res., 8, 315, 1989.

Morse, P. M., and H. Feshbach, Methods of Theoretical Physics, McGraw-Hill, New York, 1953.

Nishida, A., Ionospheric screening effect and storm sudden commencement, J. Geophys. Res., 69, 1861, 1964.

Potemra, T. A., H. Lühr, L. J. Zanetti, K. Takahashi, R. E. Erlandson, G. T. Marklund, L. P. Block, L. G. Blomberg, and R. P. Lepping, Multisatellite and ground-based observations of transient ULF waves, J. Geophys. Res., 94, 2543, 1989.

Russell, C. T., and R. C. Elphic, Initial ISEE magnetometer results, Space Sci. Rev., 22, 661, 1978.

Russell, C. T., and R. C. Elphic, ISEE observations of flux transfer events at the dayside magnetopause, Geophys. Res. Lett., 6, 33, 1979.

Sibeck, D. G., et al., The magnetospheric response to 8-minute period strong-amplitude upstream pressure variations, $J$. Geophys. Res., 94, 2505, 1989 a.

Sibeck, D. G., W. Baumjohann, and R. E. Lopez, Solar wind dynamic pressure variations and transient magnetospheric signatures, Geophys. Res. Lett., 16, 13, 1989 b.

Singer, H. J., D. J. Southwood, R. J. Walker, and M. G. Kivelson, Alfvén wave resonances in a realistic magnetospheric magnetic field geometry, J. Geophys. Res., 86, 4589, 1981.

Southwood, D. J., Localised compressional hydromagnetic waves in the magnetospheric ring current, Planet. Space Sci., 25, 549, 1977.

Southwood, D. J., Magnetopause Kelvin-Helmholtz instability, Eur. Space Agency Spec. Publ. ESA SP-148, 357, 1979.

Southwood, D. J., The ionospheric signature of flux transfer events, J. Geophys. Res., 92, 3207, 1987.

Southwood, D. J., and M. G. Kivelson, Magnetospheric interchange motion, J. Geophys. Res., 94, 299, 1989.

Southwood, D. J., and M. G. Kivelson, The magnetohydrodynamic response of the magnetospheric cavity to changes in solar wind pressure, J. Geophys. Res., 95, 2301, 1990 .

Southwood, D. J., and M. G. Kivelson, An approximate description of field-aligned currents in a planetary magnetic field, J. Geophys. Res., 95, in press, $1990 \mathrm{~b}$.

Tamao, T., A hydromagnetic interpretation of geomagnetic SSC, Rep. Ionos. Space Res Jpn., 18, 16, 1964.

M. G. Kivelson and D. J. Southwood, Institute of Geophysics and Planetary Physics, University of California, Los Angeles, CA 90024-1567.
(Received October 8, 1989; revised May 7, 1990; accepted June 8, 1990.) 